\title{
Is the informational content of histopathological reports increasing?
}

\author{
S S Cross, A D Bull
}

\begin{abstract}
Histopathology reports on 20 mastectomy and 20 colectomy specimens containing carcinomas were examined from the beginning of each decade for the period 1940-1990. The number of words and items of information in each report were recorded. There was a large (337\%) increase in the number of words in reports of both types of specimens with a slightly smaller increase $(273 \%)$ in the number of items of information. This increase may be due to clinician-led demand for more specific information or be related to the introduction of more detailed systems of pathological staging and prognostic assessment of breast and colonic tumours. The increase in data production and dissemination may not be reflected in workload measurement systems, such as Welcan, and must be considered when assessing the need for secretarial staff.
\end{abstract}

The workload of histopathological laboratories needs to be accurately measured so that resources can be used appropriately in a changing clinical environment. In histopathology the Welcan workload measurement system ${ }^{1}$ provides a more accurate assessment than the tally of the number of laboratory requests. ${ }^{23}$ The Welcan system takes account of the number of blocks, slides, and special techniques required to report a specimen but does not assess the amount of information produced in the report. If the amount of information in a report increases without an increase in the number of laboratory procedures then there could be an expansion in workload for histopathologists and secretarial staff without a concomitant rise in measured Welcan units. This study investigates whether there has been an increase in the length and informational content of histopathological reports over the past 50 years.

\section{Methods}

The diagnostic histopathological reports from a teaching hospital in Sheffield (The Royal Infirmary until 1977, then the Royal Hallamshire Hospital) were examined. During this period all reports were handwritten by pathologists and then typed by secretaries; no wordprocessing technology was used. The first 20 reports of both mastectomy and colectomy specimens containing carcinoma were studied for each decade from 1940 to 1990 . The number of words in the pathological report was recorded together with the number of items of information. An item of information was taken to be a measurement, tumour type, tumour subtype, tumour grade, comment on vascular channel invasion, comment on lymph node state, comment on depth or extent of invasion, comment on background tissue, comment on completeness of excision, formal staging and prognostic indicators. The mean number of words and items for each type of specimen was calculated for each decade.

The Mann-Whitney $U$ test was used to assess statistical significance because the distribution of results was platykurtic. The worload of the laboratories during this period was assessed using unweighted Korner requests (the information required to use the Welcan system was not available for the early years of the study).

\section{Results}

The main results are shown in figs 1 and 2. The increase in words and items between 1940 and

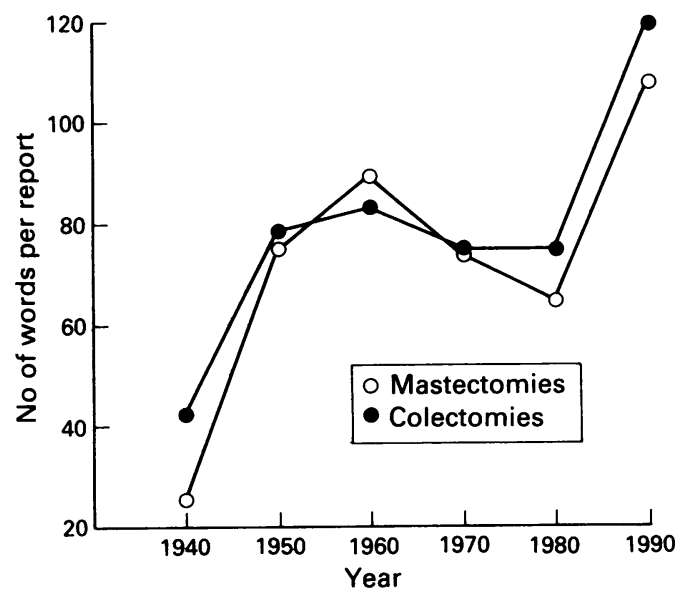

Figure 1 Mean number of words per report for malignant mastectomy and colectomy specimens.

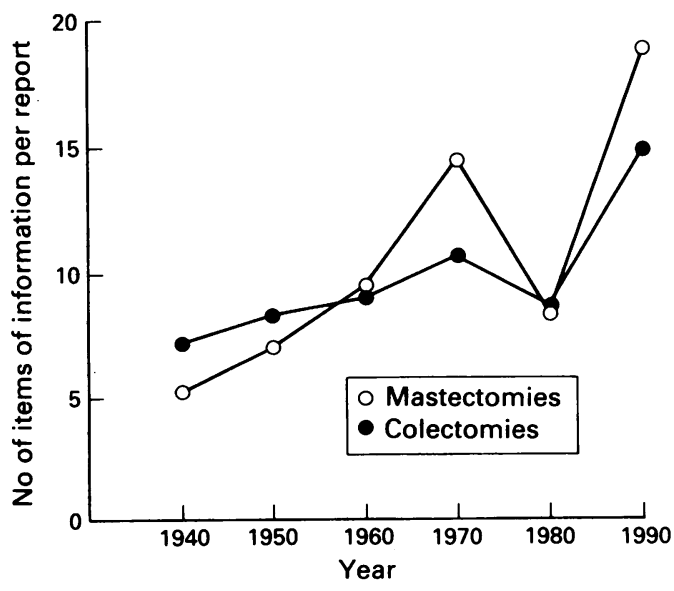

Figure 2 Mean number of items of information per report for malignant mastectomy and colectomy specimens. 
1990 reached a high level of significance ( $p<0.0001$ ) for both types of specimens. The number of laboratory requests increased from 1446 in 1940 to 14794 in 1990.

\section{Discussion}

This study shows that there has been a large increase in the number of words $(337 \%)$ and items of information (273\%) in reports of mastectomy and colectomy specimens. It is not clear whether this increase is due to demand from clinicians or a spontaneous reporting of more information by pathologists. The increases do not seem to have any temporal relation with the publication of pathological grading or staging systems. The largest increases in the number of words for both types of specimen occurred from 1940-1950 and 1980-90, with the main increase in the number of items occurring between 1980-1990. Dukes' staging of rectal carcinomas was first reported in $1932^{4}$ with a follow up study in $1958^{5}$; Jass described another system of staging in $1987,{ }^{6}$ but this was not used in the department of this study in 1990. Bloom and Richardson's method of grading breast carcinoma was published in $1957^{7}$; breast screening did not start in the centre until late 1990 and these specimens were not included in the study.

During the study period the workload of the laboratory (as measured by number of request forms) has increased by over 10 times. Although the number of staff has also increased, there has not been a reduction in the workload of an individual so the increase in report length cannot be related to this. The increase in number of words is not related to the mode of reporting because the reports have always been handwritten by the pathologists before typing. The ratio of words per item of information varies over the study period, but the figure in 1990 is only 1.3 words more than in 1940 , so there has only been a slight increase in verbosity. There has been an increase in the number of trainee pathologists in the department and they may have been writing longer reports in preparation for examinations; it would be interesting to see if the same increases have occurred in district general hospitals.

With the wide availability of word-processing equipment a long report may not take as much secretarial time to produce as a shorter report created on a typewriter. An increase in efficiency using this technology is facilitated by the development of standardised reports for certain types of specimens. In the departments included in this study word-processors were not used to prepare surgical pathology reports so any increase in report length would increase the amount of secretarial work. The Welcan system has been described only in the past decade and it was not possible to apply it retrospectively to the specimens in this study to ascertain whether the increase in words and information was reflected in an increase in Welcan units. The results of this study indicate the need to review the length and informational content of histopathological reports when assessing total laboratory workload.

1 DHSS. WELCAN Workload measurement system for path ology: manual with schedule of unit values. 1988-89 Edition. Cardiff: DHSS, Welsh Office.

2 Cavill I. Measurement of pathology workload in the United Kingdom. J Clin Pathol 1988;41:817-9.

3 Tarbit IF. Laboratory costing system based on number and type of test: its association with the Welcan workload measurement system. J Clin Pathol 1990;43:92-7.

4 Dukes CE. The classification of cancer of the rectum. $J$ Pathol Bacteriol 1932;35:323-32.

5 Dukes CE, Bussey HJR. The spread of rectal cancer and its effect on prognosis. Br J Surg 1958;12:309-20.

6 Jass JR, Morson BC. Reporting colorectal cancer. J Clin Pathol 1987;40:1016-23.

7 Bloom HJG, Richardson WW. Histological grading and prognosis in breast cancer. Br J Cancer 1957;11:359-77.

\author{
Département de \\ Pédiatrie, Hôpital des \\ Enfants-Malades, \\ Paris \\ $M$ de Montalembert \\ G Lenoir \\ A Saint-Raymond \\ J Rey \\ Institut National de \\ Transfusion Sanguine, \\ 6 rue Alexandre- \\ Cabanel 75015, Paris \\ $\mathrm{J}$-J Lefrère \\ Correspondence to \\ Dr J-J Lefrère \\ Accepted for publication \\ 31 July 1991
}

\title{
Increased PIVKA-II concentrations in patients with cystic fibrosis
}

\author{
M de Montalembert, G Lenoir, A Saint-Raymond, J Rey, J-J Lefrère
}

\author{
Abstract \\ Serum vitamin $K$ concentrations and \\ prothrombin induced by absence of \\ vitamin $K$ (PIVKA-II) concentrations \\ were assayed in 43 patients with cystic \\ fibrosis. Twenty nine showed normal \\ PIVKA-II and vitamin $K$ concentrations; \\ 14 showed an increased PIVKA-II concen- \\ tration, in one of whom serum vitamin $K$
}

was decreased. Although their vitamin $K$ concentrations were normal, some patients with cystic fibrosis still had an increased PIVKA-II. There was a significant correlation between PIVKA-II concentrations and the administration of antibiotics, a factor which has not previously been considered responsible for an increase in PIVKA-II. 